\title{
Laboratory tests for Diode-Laser based Calibration Systems for Fast Time-of-Flight Systems
}

\author{
M. Bonesini ${ }^{* 1} \nmid$ R. Bertoni ${ }^{1}$, A. DeBari ${ }^{2}$, M. Rossella ${ }^{2}$ \\ ${ }^{1}$ Sezione INFN Milano Bicocca, Dipartimento di Fisica G. Occhialini, Piazza Scienza 3, Milano, \\ Italy \\ ${ }^{2}$ Sezione INFN Pavia and Dipartimento di Fisica, Università di Pavia, via A. Bassi 6, Pavia, Italy
}

\begin{abstract}
For the time calibration of multi-channel scintillator time-of-flight detectors, a system based on commercial laser diodes in the visible range as light source and on multimode fiber patches, fused fiber splitters and optical switches, to deliver light pulses to the individual channels, may be assembled. The main problem is the tight light power budget of the system, as the available laser diodes have limited peak power. In addition, the used technology is common in the Telecom wavelength range $(\sim 850,1300-1500 \mathrm{~nm})$ but not in the visible one. Extensive laboratory tests were done on purpose and a full scale prototype calibration system was assembled at INFN Milano Bicocca.
\end{abstract}

Technology and Instrumentation in Particle Physics 2014

2-6 June, 2014

Amsterdam, the Netherlands

\footnotetext{
* Speaker.

${ }^{\dagger}$ E-mail: maurizio.bonesini@mib.infn.it
} 


\section{Introduction}

Scintillator based time-of-flight detectors may be made with arrays of individual counters, along one cartesian axis ( $x$ or $y$ ), read at one extreme by photodetectors. They may be arranged also along both orthogonal $x / y$ directions and/or be read out at both extremes, for redundancy.

Examples are the large $7.4 \times 2.5 \mathrm{~m}^{2}$ time-of-flight wall of the Harp experiment [1], using Bicron 408 scintillators and Phillips XP2020 photomultipliers, the MEG timing counter system [2], using Bicron BC 404 scintillators and Hamamatsu R5924 fine-mesh photomultipliers and the MICE time-of-flight system [3], using Bicron BC-404 or BC-420 scintillators and Hamamatsu R4998 photomultipliers. For a particle crossing a single counter $i$, with double-sided photodetector readout, the time difference $\Delta t_{i, j}$ between the STOP from the PMT $j(j=1,2)$ and the START from a reference counter $t_{s}$ is given by:

$$
\Delta t_{i, j}=t_{0}+\frac{L / 2 \pm x}{v_{e f f}}-t_{s}+\delta_{i, j} \quad j=1,2
$$

where $t_{0}$ is the particle crossing time, $x$ its distance from the counter center, $L$ is the scintillator length, $v_{\text {eff }}$ the effective light velocity in the scintillator slab $\left(v_{e f f}^{-1} \sim 6.2 \mathrm{~ns} / \mathrm{m}\right)$ and $\delta_{i, j}$ include all system delays (cables, PMT transit time, etc.). After correction for the delays $\delta_{i, j}$, the quantity

$$
\Delta t_{+, i}=\frac{\Delta t_{i, 1}+\Delta t_{i, 2}}{2}=t_{0}+\frac{L}{2 \cdot v_{e f f}}-t_{s}
$$

is independent from the particle impact point and allows the measurement of the time-of-flight (TOF), which may be used for particle identification (PID).

For good performances of a time-of-flight system it is essential the precise determination of the individual delays $\delta_{i, j}$ that may drift during the data-taking, due to temperatures excursions and other effects. With typical delays around $100 \mathrm{~ns}$, time drift of the order of 9 (3) ps/ $/{ }^{\circ} \mathrm{C}$ may be reached for RG58 (RG213) signal cables. This must be compared with a TOF detctor resolution $\left(\sigma_{t}\right)$ in the range $50-150 \mathrm{ps}$.

Detector timing calibration in a time-of-flight system means the precise determination of delays $\delta_{i, j}(t)$ at an initial time $t_{0}$ and the monitoring of their value along the data-taking. This may be done with cosmic rays or impinging particles, if available: muons in the case of MICE, or by delivering a fast calibration pulse to each individual channel. The requirement on the calibration pulses is that their rising edge mantains the original characteristics, with minimimal deterioration, as respect to the one delivered by the used calibration light source.

Previous systems include the ones used for the $N-\bar{N}$ experiment at Grenoble [4], the MARKIII experiment at SLAC [5], the CLAS system at CEBAF [6], the TOPAZ experiment at KEK [7] and the HARP/PS214 experiment at CERN [8].

These systems are based on a fast light source, typically a laser with FWHM 30-50 ps, and an optical system to deliver the laser pulses to each individual channel, to be calibrated. As an example, in reference [8] a duplicated Nd/Yag laser at $532 \mathrm{~nm}$, with passive Q-switch and active/passive mode locking was used ${ }^{1}$, followed by an optical delivery system to the individual

\footnotetext{
${ }^{1}$ model SYLP0 from Quanta Systems srl, Italy, with 60 ps FWHM, $10 \mathrm{~Hz}$ repetition rate, $3 \mathrm{~mJ}$ energy per pulse
} 
scintillators, based on a bundle of 64 Corning SMF-28 IR monomode fibers ${ }^{2}$. Systems of this type are expensive and quite difficult to operate.

The use of low-cost laser-diode systems, but unfortunately also limited power, has been proposed inside the T0 detector group of the ALICE Collaboration at LHC [9]. This kind of lasers have high repetition rates (up to several $\mathrm{MHz}$ ) but lower energies per pulse (up to a factor $10^{-6}$ lower), putting severe constraints on the optical laser pulse delivery system to the individual channels.

\section{Tests of optical system components}

A laser diode calibration system may be built up from optical switches that direct the input laser pulse to one of $N$ output channels, fused fiber splitters $1 \times N$ that divide the input laser pulse to $N$ output channels and fiber patch cables for connections between the previous items.

In all optical elements the laser pulse must be transmitted with minimimal attenuation and a minimal deterioration of its timing characteristics, mainly its risetime. Assuming a gaussian shape for the impulse, the key parameter for characterisation of such waveform is its standard deviation $\sigma$ ${ }^{3}$. The setup used for the measurement of the tested optical components is shown in figure 2. Light from a fast laser ${ }^{4}$ is injected into a MM Arden Photonics ModCon mode scrambler ${ }^{5}$ (or a $1 \mathrm{~m}$ long OZ/OPTICS IRVIS fiber) using a Newport 20x objective. Fine alignment for the injection is obtained via a $x, y, z$ Newport micrometric stage. After the last optical element under test, the ouput light pulse is focalized, via another 20x objective on a micrometric $x, y, z$ flexure stage, on a fast MSM Hamamatsu G4176 photodetector (with 30 ps rise and fall time). For optimal performances, the photodetector is powered by a $10 \mathrm{GHz}$ broadband Picosecond Pulse Lab 5550B bias tee and its signal is then amplified by a $10 \mathrm{~dB}$ broadband inverting amplifier ${ }^{6}$. The amplified signal is then fed into a $20 \mathrm{GHz}$ HP 54750 sampling scope. The trigger signal is given by the laser sync out. The used laser source (Pilas 040 from Advanced Laser Systems) has been characterized by the manufacturer with a $20 \mathrm{GHz}$ Ultrafast detector and a $50 \mathrm{GHz}$ HP54750A scope ( a typical measurement is shown in figure 1). In our test system, with only the injection system inserted, we may expect a signal FWHM given by:

$F W H M_{0}=\sqrt{F W H M_{\text {laser }}^{2}+F W H M_{\text {bias tee }}^{2}+F W H M_{\text {scope }}^{2}+F W H M_{G 4176}^{2}} \leq 81$ ps $(\simeq 73$ ps typical $)$

where the FWHM are $\sim 35$ ps for the laser, $\sim 30$ ps for the bias tee, $\sim 22$ ps for the HP54750 scope, $\leq 55 \mathrm{ps}$ ( $\simeq 42$ ps typical) for the G4176 MSM photodetector ${ }^{7}$ and $\sim 30$ ps for the fast amplifier.

This number has to be compared with a measured value of $80.5 \pm 0.3$ ps $(78.7 \pm 0.1 \mathrm{ps})$ with an Arden Photonics mode scrambler (1m of OZ/OPTICS IRVIS fiber) inserted.

\footnotetext{
${ }^{2}$ made by Fiberlan srl, Milano

${ }^{3} \mathrm{We}$ remind here for easiness the main relations between often measured time parameters in this gaussian approximation: $F W H M=2.354 \times \sigma$, risetime $(10-90 \%)=1.6888 \times \sigma=$ falltime $(10-90 \%)$

${ }^{4}$ Pilas 040 from Advanced Laser Systems, with FWHM $\sim 30 \mathrm{ps}$, repetition rate up to $1 \mathrm{MHz}$, peak power $1 \mathrm{~W}$, free space beam head optics

5 introduced here to create more uniform laser launch conditions, to reduce measurement variability

${ }^{6}$ Model BBA-3 from Alphalas Gmbh, with $15 \mathrm{GHz}$ bandwidth

${ }^{7}$ datasheet FWHM data for G4176 photodetectors from the manufacturer are in the range $70-80$ ps with a similar test system
} 


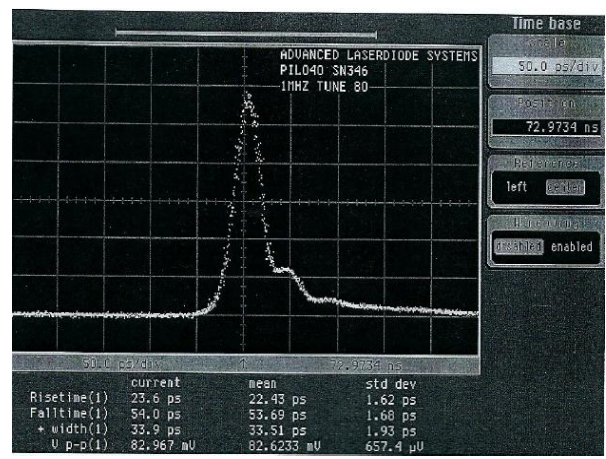

Figure 1: Measure of the timing characteristics of the used Pilas 040 laser, with an HP54750A sampling scope

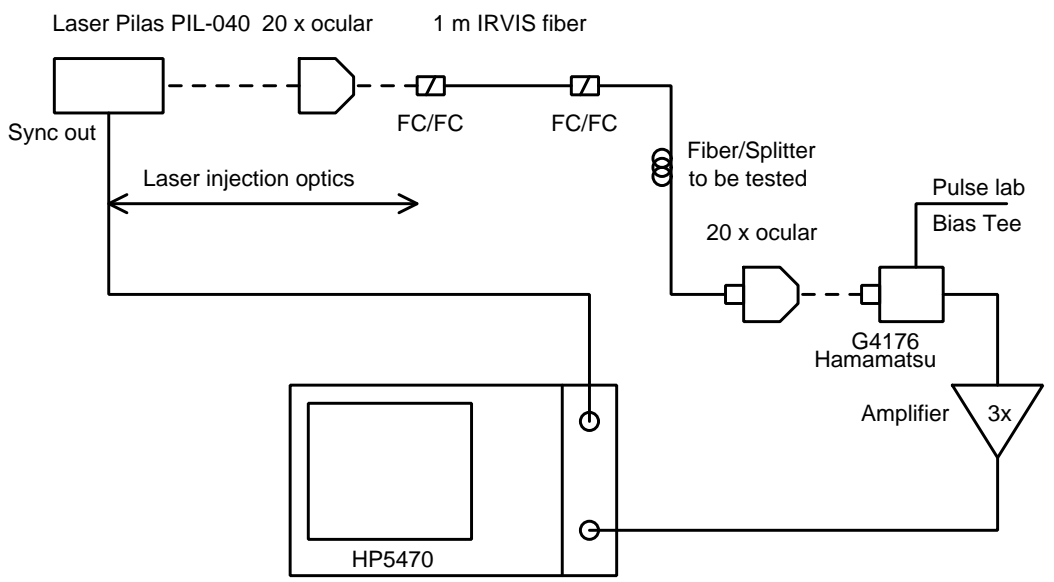

Figure 2: Experimental setup for test of single components, such as fiber patches, fused fiber splitters and optical switches. In the attenuation studies the Hamamatsu photodetector+HP54750 sampling scope was replaced by a power meter

Timing characterization of single optical components, such as fiber patch cords, optical switches, fused fiber splitter, has been obtained by putting, after the insertion stage, the elements to be tested and measuring the increase in the signal FWHM $\left(\triangle F W H M=\sqrt{F W H M^{2}-F W H M_{0}^{2}}\right)$.

\subsection{Tests of used optical fibers}

To guarantee an optimal and simple injection of the light from the laser source, large core multimode fibers (MM) are to be preferred to small core single mode (SM) fibers. The problem is that MM fibers may give a remarkable deterioration of the timing properties of the propagating laser pulse. This has to be checked in the real experimental conditions on the distances that may be of relevance to the calibration setup, e.g. with fiber patchcords up to 15-20 meters, to go from the laser source to the detector channels to be calibrated. Tests were done with the setup of figure 2 

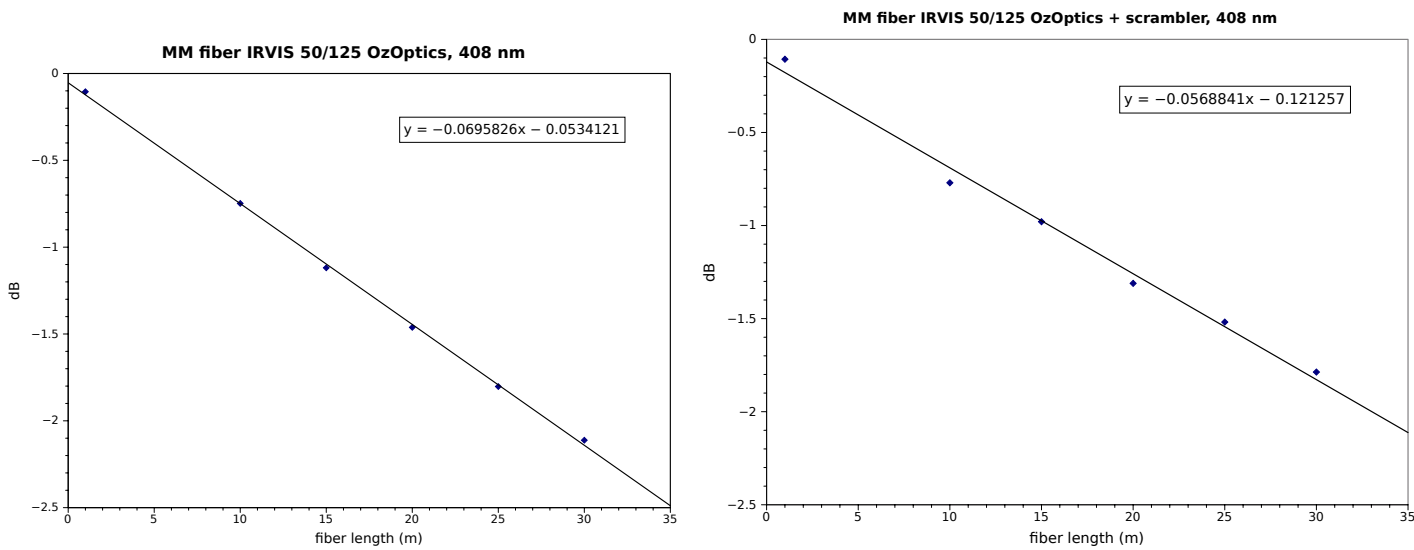

Figure 3: Attenuation in dB for OZ/OPTICS IRVIS 50/125 MM fibers. Left panel: without mode scrambler; right panel: with mode scrambler.

where the signal after the fiber under test was measured directly by a power meter ${ }^{8}$ for attenuation studies and by the full setup with a Hamamatsu G4176 photodetector and a HP54750 sampling scope for the timing studies. Attenuation studies were done with and without the optical mode scrambler and are resumed in table 1.

Table 1: Attenuation and timing properties of measured MM fibers.

\begin{tabular}{|c|c|c|c|}
\hline MM fiber & fiber type & $\begin{array}{l}\text { Attenuation }(\mathrm{dB} / \mathrm{m}) \\
\text { (no scrambler) }\end{array}$ & $\begin{array}{l}\text { Attenuation }(\mathrm{dB} / \mathrm{m}) \\
\text { (scrambler) }\end{array}$ \\
\hline Thorlabs AFS 50/125Y & $\begin{array}{c}\text { step index: } 400-2400 \mathrm{~nm} \\
50 \mu \mathrm{m} \text { core }\end{array}$ & $0.169 \pm 0.007$ & $0.193 \pm 0.011$ \\
\hline Thorlabs AFS 105/125Y & $\begin{array}{c}\text { step index: } 400-2400 \mathrm{~nm} \\
100 \mu \mathrm{m} \text { core }\end{array}$ & $0.0796 \pm 0.0026$ & $0.0808 \pm 0.0046$ \\
\hline Thorlabs SFS 50/125Y & $\begin{array}{l}\text { step index: } 250-1200 \mathrm{~nm} \\
50 \mu \mathrm{m} \text { core }\end{array}$ & $0.0657 \pm 0.0052$ & $0.0744 \pm 0.0097$ \\
\hline Corning 50/125 & $\begin{array}{l}\text { graded index } \\
50 \mu \mathrm{m} \text { core }\end{array}$ & $0.0794 \pm 0.0029$ & $0.0894 \pm 0.0051$ \\
\hline OZ/OPTICS 50/125 IRVIS & $\begin{array}{c}\text { graded index: } 400-1800 \mathrm{~nm} \\
50 \mu \mathrm{m} \text { core }\end{array}$ & $0.0696 \pm 0.0026$ & $0.0569 \pm 0.00063$ \\
\hline OZ/OPTICS 50/125 UVVIS & $\begin{array}{l}\text { step index: } 200-900 \mathrm{~nm} \\
50 \mu m \text { core }\end{array}$ & $0.118 \pm 0.009$ & $0.114 \pm 0.007$ \\
\hline
\end{tabular}

Figure 3 shows the attenuation measured with a graded index multimode OZ/OPTICS IRVIS 50/125 fiber, using patches of different lengths. Results on pulse dispersion, in terms of pulse delay and increase in the 10-90\% risetime or in the FWHM are shown in figure 4 for OZ/OPTICS IRVIS 50/125 fibers. All tests were done with a mode scrambler before the fiber patch to be tested.

As the variation of delays $\delta_{i}$ is mainly due to thermal excursions in the experimental hall housing the used TOF system, it is important to study also the influence of temperature on the used

${ }^{8}$ Ophir Nova with a PD300 head 

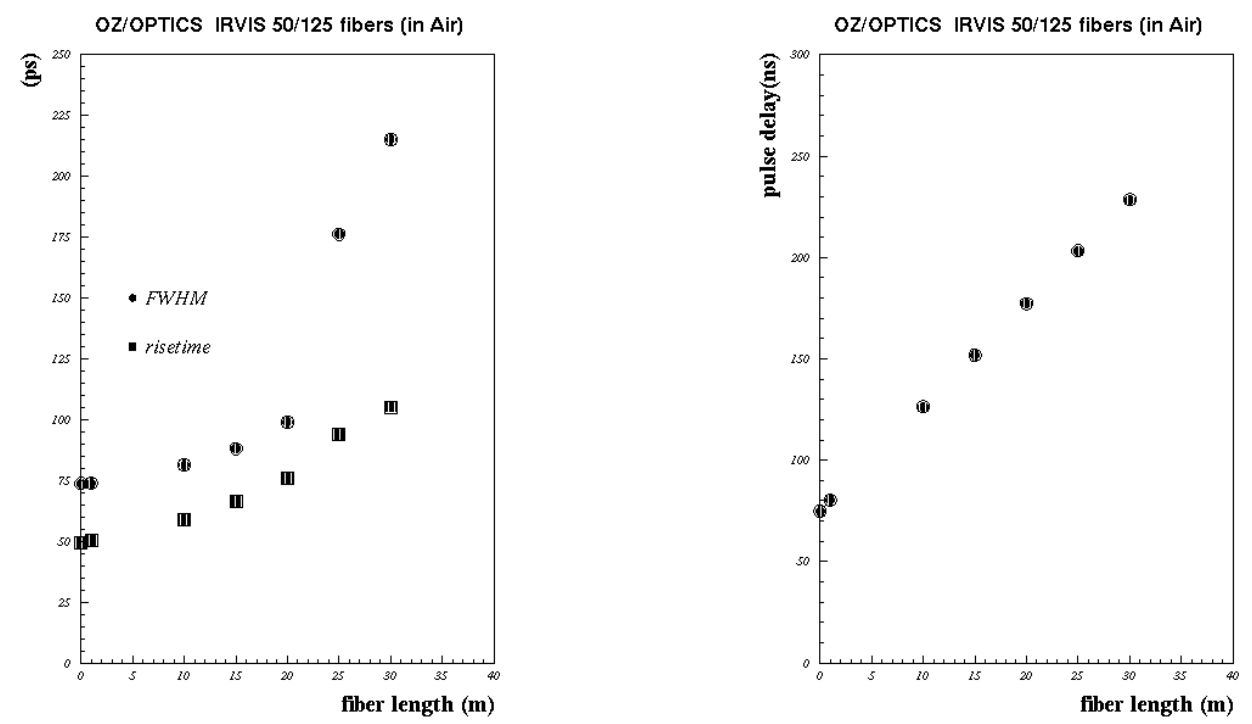

Figure 4: Left panel: Increase in the FWHM or the 10-90 \% risetime inserting a fiber patch cord of length $\mathrm{L}$ after the input insertion mode scrambler. Used fiber is $50 / 125 \mu \mathrm{m}$ OZ/Optics IRVIS. Right: pulse delay for the same fiber patches.
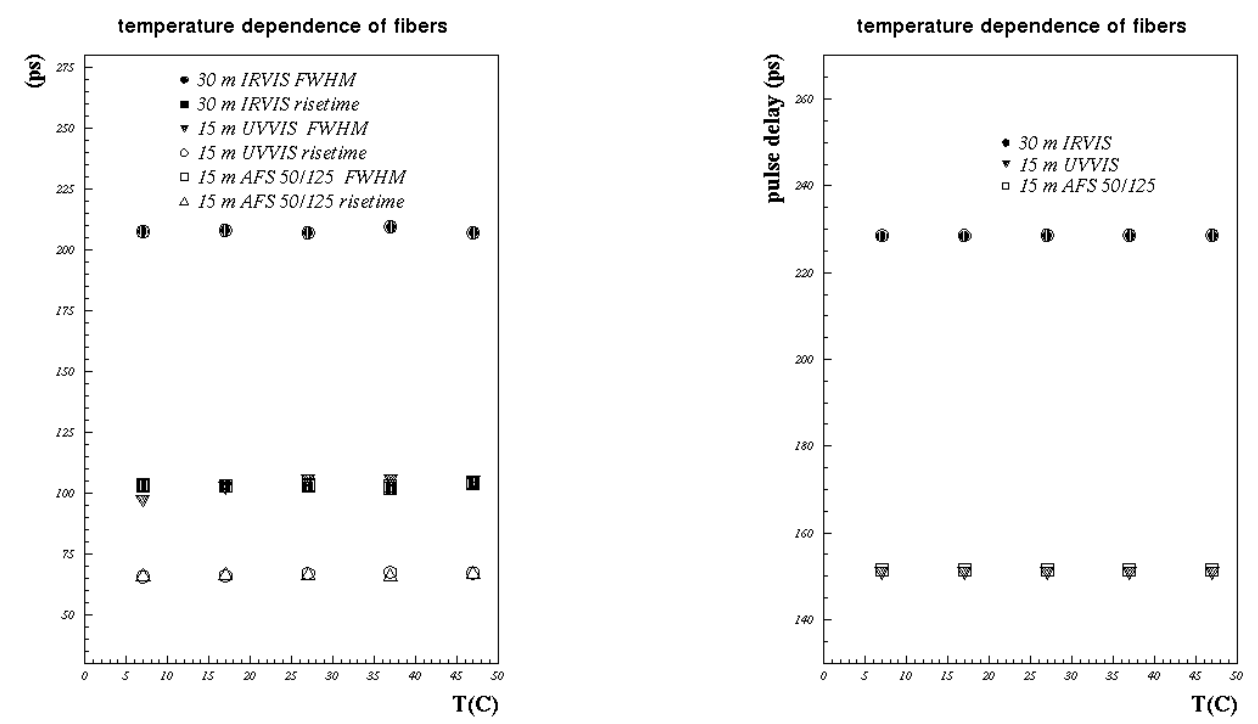

Figure 5: Temperature dependence for different MM fiber patches. Left panel: FWHM or 10-90\% risetime vs temperature. Right panel: pulse delay vs temperature.

fiber patches. A precision LAUDA cooling thermostat RP845 (precision $\pm 0.01{ }^{0} \mathrm{C}$ of the thermal bath), where a part of the fiber under test was kept at fixed temperature, was added to our test system. Three patches were tested: two $15 \mathrm{~m}$ Thorlabs AFS 50/125 and OZ/OPTICS UVVIS fiber patches (14 $\mathrm{m}$ in the thermal bath, $1 \mathrm{~m}$ in air) and one $30 \mathrm{~m}$ OZ/OPTICS IRVIS 50/125 fiber patch ( $28 \mathrm{~m}$ in the thermal bath, $2 \mathrm{~m}$ in air). Results are shown in figure 5 and no influence is seen in the operating range between 7 and $50{ }^{\circ} \mathrm{C}$. As a conclusion, the chosen OZ/OPTICS IRVIS $50 \mu \mathrm{m}$ fiber 
has a pulse width dispersion $\sim 1 \mathrm{ps} / \mathrm{m}$, an attenuation $\sim 0.06 \mathrm{~dB} / \mathrm{m}$ and its delay is $\sim 5.11 \mathrm{~ns} / \mathrm{m}$ with a temperature effect of $0.2 \%$ assuming a temperature excursion of $10{ }^{0} \mathrm{C}$ for a $10 \mathrm{~m}$ fiber.

\subsection{Tests of optical switches and fused fiber splitters}

Optical switches direct the input signal to one of $N$ outputs with minimal insertion losses. The maximum number of available output channels is typically 16-32 in the visible range, using MM fibers. Optical fiber fused splitters instead split the input signal over $N$ ouput channels in an even way (if required). These last components are quite common for the Telecom range of wavelengths (850 $\mathrm{nm}$ or 1300-1500 $\mathrm{nm}$ ) but difficult to obtain for the visible range at $\sim 400 \mathrm{~nm}$.

A custom-made MM optical switch $1 \times 9$ by PiezoJena ${ }^{9}$ and several custom-made optical splitters $1 \times 2,1 \times 4,1 \times 8$ by OZ/Optics and Lightel were tested.

After the mode scrambler, the measured FWHM of the laser signal increased from $80.47 \pm 0.61$ ps to typical values around $83.14 \pm 0.46 \mathrm{ps}$ inserting the optical switch. The output signal variation from channel to channel was within $1 \%$, with a cross-talk $\sim 2 \%$ and an insertion loss $\sim 40 \%$. These numbers are to be compared with manufacturer specs, albeit at $\lambda=850 \mathrm{~nm}$, where an insertion loss of $1.5 \mathrm{~dB}$ and a cross-talk $\sim 70 \mathrm{~dB}$ are quoted.

Similar measurements were done inserting optical fused fiber splitters in place of the optical switch, after the mode scrambler. We see a relevant insertion loss at $400 \mathrm{~nm}$ (around 2-3 dB) and a spread in the splitting ratio $\sim 10-15 \%$. The measured FWHM of the input laser signal increased typically of $2-3$ ps after insertion of the optical splitters. Results for the tested even fiber splitters are shown in table 2.

Table 2: Measured properties of MM fused fiber splitters from OZ/Optics and Lightel Ltd. (A), (B), (C), (D), (E), (F), (G) refer to different specimens. (A), (B), (F), (G) have SMA output connectorized pigtails, while (C), (D), (E) have FC connectors. The input pigtail has always an FC connectors.

\begin{tabular}{|c|c|c|}
\hline & $\begin{array}{c}\text { spread in output splitting } \\
\text { as rms/average }(\%)\end{array}$ & tot. insertion loss $(\mathrm{dB})$ \\
\hline OZ/Optics (A) $1 \times 4$ & 9.5 & $2.2 \mathrm{~dB}$ \\
OZ/Optics (B) $1 \times 4$ & 6.2 & $3.9 \mathrm{~dB}$ \\
OZ/Optics (C) $1 \times 4$ & 15.2 & $2.5 \mathrm{~dB}$ \\
Lightel (D) $1 \times 4$ & 3.2 & $3.2 \mathrm{~dB}$ \\
Lightel (E) $1 \times 4$ & 6.7 & $3.0 \mathrm{~dB}$ \\
Lightel (F) $1 \times 4$ & 8.5 & $3.0 \mathrm{~dB}$ \\
Lightel (G) $1 \times 8$ & 11.5 & $3.2 \mathrm{~dB}$ \\
\hline
\end{tabular}

In conclusion, the tested optical components: switches, fused fiber splitters, fiber patchcords seem all suitable to assemble the pulse delivery system for a diode-laser based calibration system.

\section{Test of a prototype calibration system}

A prototype calibration system has been assembled in laboratory at INFN Sezione Milano Bicocca from the previous optical elements. In its present configuration, up to 36 (72) individual

\footnotetext{
${ }^{9}$ Model F-109-05 with Thorlabs SFS 50/125 fibers as pigtails
} 


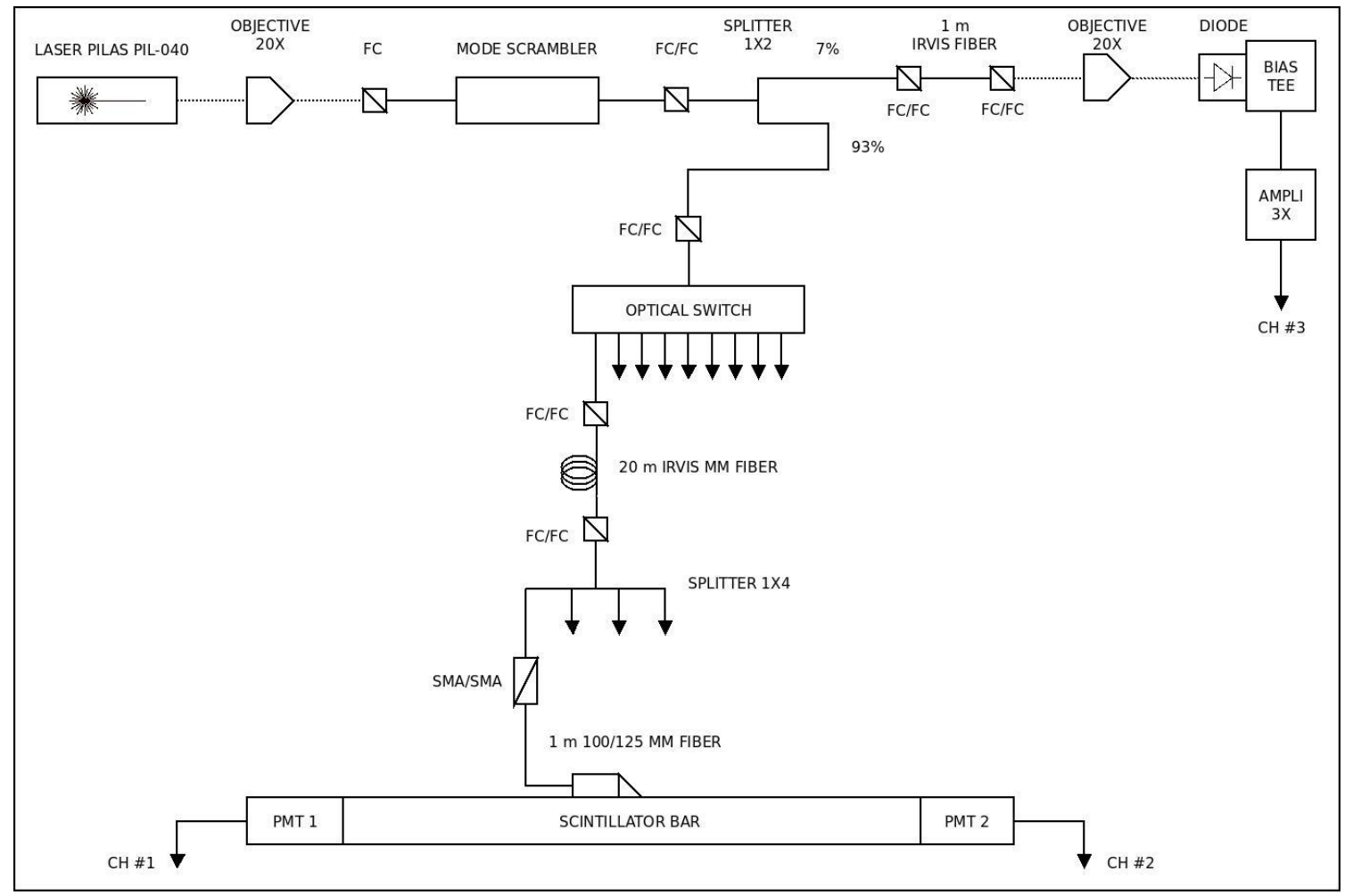

Figure 6: Schematic layout of the tested prototype calibration system.

channels (using $1 \times 4$ or $1 \times 8$ splitters) may be calibrated. It may easily extended to configurations with up to 100-200 channels. Configurations with more channels require more powerful diodelasers systems, that are difficult to find now on the market or a more tight control on the power budget of the system. Figure 6 shows the setup presently assembled in laboratory, where the light from one $1 \times 4$ splitter is injected, by means of a reflection prism, into the center of the scintillation counter to be calibrated.

With a trigger on cosmic rays, put at the center of the scintillation counter, it was possible to see that the calibration signal in the counter is roughly a factor 2-3 bigger than the one of the cosmic muons.

The PMT signal were acquired with a VME system, based on the CAEN V2718 interface, and splitted by a 50\% passive splitter to a CAEN V792 QADC and a CAEN V1290 TDC, after a leading edge discriminator. TDC signals are computed as differences between the L/R PMT signal and a reference signal sent, after the 1-2 splitter, to a fast photodiode ${ }^{10}$. Care was taken to have the intensity of the direct signal comparable to the one after the calibration system, by using optical attenuators.

Results are compatible and show that the calibration system does not introduce unbearable attenuation losses or timing deterioration of the calibration signals.

\footnotetext{
${ }^{10}$ Thorlabs DET02A, rise time $50 \mathrm{ps}$ and fall time $150 \mathrm{ps}$
} 


\section{Conclusion}

Optical components to assemble a calibration system, based on a laser diode as a light source, have been extensively tested. Optical switches and fused splitters introduce minimal deterioration on the timing properties of the delivered laser pulse. The same is true for optical fiber patches, if their length is less than 10-15 meters. A prototype system has been assembled in laboratory at INFN Sezione Milano Bicocca. We conclude that such a calibration system may be used for fast scintillator based TOF system, with up to 100-200 channels and timing resolutions in the range 50-100 ps, such as the one developed for the MICE experiment at RAL [10]

\section{Acknowledgements}

We would like to acknowledge the skilfull work of Mr. R. Mazza, F. Chignoli INFN Milano Bicocca for the realization of the test setup and thank Dr. L. Mariani of dB Electronics for helpful discussions.

\section{References}

[1] Baldo Ceolin, M. et al. , "The time-of-flight TOFW detector of the HARP experiment: Construction and Performance", Nucl. Instr. Meth. A532 (2004) 548.

[2] Dussoni, S. et al., "The Timing Counter of the MEG Experiment: Design and Commisioning", Nucl. Instr. Meth. A617 (2010),387.

[3] Bertoni, R. et al., "The design and commissioning of the MICE upstream time-of-flight system", Nucl.Instrum.Meth. A615 (2010) 14.

[4] Baldo Ceolin, M. et al., Performance of the $\mathrm{N}-\bar{N}$ Scintillation counters Trigger and TOF System, Nuovo Cimento 105A (1992) 1679.

[5] Brown, J.S. et al., "The Mark III time-of-flight system", Nucl. Instr. Meth. 221 (1984),503

[6] Smith, E.S. et al., "The time-of-flight system for CLAS", Nucl. Instr. Meth. 432 (1999) 265.

[7] Kishida, T. et al., "A laser calibration system for the KEK TOPAZ barrel TOF counters", Nucl. Instr. Meth. 254 (1987) 367.

[8] Bonesini, M. et al., "Construction of a Fast Laser-based Calibration System for the Harp TOF counters Wall", IEEE Trans. Nucl. Sci NS-50 (2003) 1053

[9] Bondila, M. et al., " ALICE T0 Detector", IEEE Trans Nucl. Sci NS-52 (2005) 1705.

[10] Gregoire, G. et al.,"MICE Technical Report", 2005, Rutherford Appleton Laboratory". 